\section{LE TOURISME RÉFLEXIF, UN NOUVEAU FONDEMENT D'UN TOURISME DURABLE}

\author{
Rémy Knafou \\ Université Paris 1 - Panthéon-Sorbonne \\ ORCID iD: http://orcid.org/0000-0002-2109-0688 \\ remy.knafou@orange.fr
}

Cómo citar este artículo/Citation: Knafou, R. (2017). Le tourisme réflexif, un nouveau fondement d'un tourisme durable. Arbor, 193 (785): a395. doi: http://dx.doi.org/10.3989/ arbor.2017.785n3001

Recibido: 23-10-2016. Aceptado: 8-6-2017.

RÉSUMÉ: Le développement du tourisme durable se heurte à la difficulté de mettre en actes un discours contradictoire, qui plus est dans un contexte où les grandes concentrations touristiques sont en place et où les flux touristiques s'intensifient chaque jour. L'une des manières d'y viser est de penser sur les pratiques des touristes. Et l'une des manières d'y parvenir est de favoriser les postures réflexives des touristes, dans le cadre d'un tourisme qu'on propose de nommer "tourisme réflexif".

MOTS CLÉS: Tourisme réflexif; tourisme durable; système touristique; mémorial; objets urbains; pratiques du tourisme; sérendipité.

ABSTRACT: The development of sustainable tourism is facing the difficulty of applying a contradictory discourse, especially when this occurs in the context of higher tourist concentrations and where the tourist flows are becoming increasingly intense. One of the ways to approach sustainable tourism and its context is to consider tourism practices. Moreover, one of the ways to reach its objectives is to encourage an attitude of reflection among tourists within the context of so-called reflective tourism.

\section{REFLEXIVE TOURISM, A NEW BASIS OF SUSTAINABLE TOURISM}

Copyright: (C) 2017 CSIC. Este es un artículo de acceso abierto distribuido bajo los términos de la licencia Creative Commons Attribution (CC BY) España 3.0.

RESUMEN: El desarrollo del turismo sostenible se enfrenta con la dificultad de utilizar un discurso contradictorio, y más en un contexto en el que se dan grandes concentraciones turísticas y en el que los flujos turísticos se intensifican de día en día. Uno de los modos de acercarse al turismo sostenible es pensar en las prácticas de los turistas. Y una de las vías para alcanzar sus objetivos es favorecer la postura reflexiva de los turistas, en el marco de un turismo que proponemos llamar turismo reflexivo.

PALABRAS CLAVE: Turismo reflexivo; turismo sostenible; sistema turístico; memorial; objetos urbanos; prácticas turísticas; serendipia.

KEYWORDS: Reflexive tourism; sustainable tourism; tourism; memorial system; urban objects; tourism practices; serenpidity. 


\section{INTRODUCTION}

Dès sa fondation, en 1993, au sein de l'université Paris 7, l'équipe MIT (Mobilités, Itinéraires, Territoires ${ }^{1}$ ), avait jeté les bases d'une démarche réflexive appliquée au tourisme en posant que le touriste est un acteur qui fait des choix et n'est pas simplement manipulé par un système touristique uniquement mû par la logique du marché. La réflexivité n’a du reste jamais fait défaut au touriste, même si celui-ci la pratiquait sans le savoir comme monsieur Jourdain faisait de la prose sans le savoir ${ }^{2}$. En revanche, tous ceux qui l'ont niée ou sous-estimée, des chercheurs aux professionnels du tourisme, ont tout intérêt désormais à s'en emparer. Régulièrement, certains se demandent comment réinventer le tourisme, à travers ateliers, colloques et séminaires, alors que les touristes passent leur temps à le faire, en développant sans cesse des pratiques nouvelles, en interaction croissante, il est vrai, avec le marché, ses destinations nouvelles et ses applications. Comme l'écrit Ph. Violier (2007), " Internet libère le touriste ", l'auto-organisation progressant sans arrêt et intervenant aussi désormais sur des destinations lointaines et exotiques vers lesquelles seuls les backpackers se hasardaient encore il y a peu. Seules les générations les plus âgées et les actifs ne disposant pas de temps pour préparer un voyage persistent à utiliser des outils que les plus jeunes contournent, tels que les agences de voyage. On sait bien que la réflexivité n'est jamais un développement solitaire, mais s'inscrit dans un processus social par lequel, à un moment donné, divers acteurs se rassemblent autour de leur compréhension mutuelle d'une situation et la manière d'y faire face.

Le tourisme n'échappe donc pas à ce besoin comme à cette recherche de réflexivité, évolution utile pour donner du sens à ce qui se joue dans la pratique touristique et qui, comme toute activité humaine, répond à des intentions comme à des besoins. L'être touriste n'est pas une espèce différente de l'Homo sapiens, il n'en est que l'une des nombreuses facettes, ce qui lui permet en particulier de concevoir des projets touristiques plus ou moins complexes, à la fois réponse à des besoins personnels et sociétaux et outils de mise en relation avec les autres et le vaste monde. Donner du sens à ses actions est une spécificité de l'espèce humaine ; avec les processus mobilisés dans le cadre du tourisme réflexif, le touriste y participe à certains moments, ce qui ne l'empêchera nullement à d'autres moments de se mettre en roue libre et, ce faisant, de prêter le flanc à des appréciations aussi sommaires que répandues dans la critique du touriste et d'un tourisme qui ne serait pas « durable ».
Or, la mise en œuvre de la réflexivité dans le domaine du tourisme s'inscrit dans la logique d'une recherche de durabilité bien comprise, qui ne se borne pas à susciter de la mauvaise conscience chez les touristes qui n'agiraient pas comme il se doit selon certains beaux esprits ; et cette mise en œuvre justifie qu'on aborde ici les relations entre ce que nous proposons d'appeler " tourisme réflexif » et ce qu'il est devenu commun de nommer « tourisme durable ».

Je ne reviendrai pas ici sur la dimension pathétique que l'attente de durabilité signifie pour nos sociétés confrontées à une accélération sans précédent de tous les facteurs de changements et, donc, de déstabilisation sur tous les plans, technologiques, sociétaux, économiques, organisationnels, moraux, etc. Je me contenterai de constater que ce choix, très contestable, de traduire en français ${ }^{3}$ l'anglais " sustainable » par « durable » est à l'origine d'une ambiguïté et d'une approximation, aussi préjudiciables l'une que l'autre dans le champ du tourisme. L'emploi du mot « durable » induit un glissement de sens pernicieux : en effet, est « durable » ce qui est de nature à durer longtemps, qui est susceptible de résister à l'épreuve du temps. Or, le " tourisme durable » est une forme de tourisme, assez mal définie du reste (cf. infra), qui suppose que pour être « durable » le tourisme doive remplir un certain nombre de conditions difficiles à réunir.

\section{QUELQUES REFLEXIONS PRELEMINAIRES SUR LE TOURISME « DURABLE »}

\subsection{La difficile " soutenabilité " d'une activité objectivement " durable »}

Le tourisme est d'abord durable - dans sa dimension historique - parce qu'il existe sur la longue durée, depuis plus de deux siècles et fait vivre des lieux de manière plus pérenne que l'industrie moderne née en même temps que lui (Equipe MIT, 2011). Mais cette durabilité-là a été occultée parce que, en français, on a préféré appeler "durable " ce qu'on souhaiterait voir durer mais qui n'est pas sûr pour autant de durer, en oubliant le sens premier et véritable du mot "durable ${ }^{4}$. Le tourisme "durable » est partout désormais, du moins dans les discours, les politiques, les offres commerciales. Sa mise en œuvre est une autre affaire, longue, difficile et généralement pas à l'échelle des problèmes à traiter. Mais, pour en juger, il convient d'en rappeler la définition la plus répandue, due à l'Organisation mondiale du tourisme : le "Développement touristique durable satisfait les besoins actuels des touristes et des régions d'accueil tout en protégeant et en améliorant les perspectives pour 
l'avenir. Il est vu comme menant à la gestion de toutes les ressources de telle sorte que les besoins économiques, sociaux et esthétiques puissent être satisfaits tout en maintenant l'intégrité culturelle, les processus écologiques essentiels, la diversité biologique, et les systèmes vivants. ". II est évident que cette définition vise à une situation idéale que les contradictions compromettent ; mais il n'en demeure pas moins qu'elle a le mérite de fixer des objectifs souhaitables dont les différents acteurs du système gagneraient à tenter de se rapprocher, à la faveur de démarches réfléchies et concertées. Or, pour se rapprocher de ces objectifs, le tourisme se heurte à une difficulté fondamentale, qui résulte précisément de sa durabilité historique, celle de ne pouvoir continuer à se développer sur un même mode, dès lors qu'il change à la fois d'échelle et de sociologie, car c'est le tourisme des très grands nombres qui évidemment pose problème et va en poser bien plus dans l'avenir, comme du reste toutes les activités humaines indexées sur la croissance démographique de notre planète.

\section{Schématiquement, trois grandes périodes}

Très schématiquement, on peut distinguer trois grandes périodes dans la mise en place du système touristique actuel.

- La période pionnière de la découverte : les premiers touristes, en arrivant dans des lieux qui ne les attendaient pas, y ont produit des effets profonds, qui ont contributé à déstructurer les sociétés locales et à les faire entrer dans des processus nouveaux et irréversibles. Peu nombreux, ces premiers touristes n'en ont pas moins entraîné des transformations considérables, de la société comme de la nature des lieux investis, des transformations souvent minimisées dans une littérature scientifique qui eut tendance à mettre l'accent sur l'étape suivante qui fut celle des changements induits par la multiplication du nombre des touristes qu'on appela " tourisme de masse » [turismo de masas].

- Le tourisme de masse caractérisa la période allant du milieu du XIXe siècle (avec les premiers voyages de groupes organisés par Thomas Cook) au dernier quart du XX $X^{\mathrm{e}}$ siècle; elle s'accompagna d'une diffusion des régions et sites touristiques dans la plus grande partie du Monde (Equipe MIT, 2011).

- La fin du XXe siècle et le début du XXI ${ }^{\mathrm{e}}$ nous a fait entrer dans le tourisme des masses [turis- mo de las masas] avec, à la fois, un nouveau changement d'échelle, une individualisation croissante des pratiques en grande partie liée à la révolution de l'Internet et une affirmation de la multipolarisation des flux d'émission de touristes. En effet, à l'ère du tourisme de masse, l'Europe principalement et l'Amérique du nord secondairement étaient les pôles émetteurs de touristes, vers leurs propres territoires comme vers une grande partie du Monde. Aujourd'hui, I'Asie supplante l'Europe, Chine en tête désormais, et presque plus aucun point de la Terre échappe à des flux inégalement importants, mais capables en très peu de temps de saturer les lieux les plus inacessibles ou se prêtant le moins à l'arrivée de grands nombres. Et dans ce processus, le tourisme " durable » a aussi une responsabilité directe.

\subsection{Des flux à une échelle sans précédent qui posent des problèmes nouveaux}

L'impossibilité de se développer sur un même mode est un fait établi depuis longtemps mais dont nos élites n'arrivent pas à faire le deuil. Chaque génération qui passe a eu une expérience propre des lieux touristiques, différente de celle qui la précédait comme de celle de la suivante. En juillet 1843, Victor Hugo évoquait ainsi Biarritz : " On se baigne à Biarritz comme à Dieppe comme au Havre comme au Tréport mais avec je ne sais quelle liberté que ce beau ciel inspire et que ce doux climat tolère [...] Je n'ai qu'une peur ; c'est qu'il devienne à la mode. Déjà l'on y vient de Madrid ; bientôt on y viendra de Paris [...] Alors, Biarritz, ce village si agreste, si rustique et si honnête encore, sera pris du mauvais appétit de l'argent. Bientôt Biarritz mettra des rampes à ses dunes, des escaliers à ses précipices, des kiosques à ses rochers, des bancs à ses grottes. Alors Biarritz ne sera plus Biarritz; ce sera quelque chose de décoloré et de bâtard comme Dieppe et Ostende » (Cerda, 2006, p. 8). On peut louer Hugo sur sa clairvoyance quant à l'avenir de Biarritz - qui fit la fortune des entrepreneurs comme des spéculateurs avisés. Mais on peut être plus critique sur ses motivations, conservatrices - la crainte, très commune, de voir un lieu qui plaît se transformer et ne plus être comme avant - et élitistes - le souhait de ne pas partager un lieu avec d'autres. Pour prendre un exemple personnel, j'ai pu, dans ma jeunesse (années 1960), jouer au tennis dans un hôtel de Chamonix ou de Zermatt. Aujourd'hui, dans des vallées qui n'ont plus de réserve foncière, il y a belle lurette que les courts de tennis ont disparu pour faire place à du bâti, plus lucratif. Dans ces mêmes lieux, j'ai pu monter au Gornergrat comme à la mer de Glace dans des trains non bondés et 
sans touristes asiatiques, comme j'ai pu entrer au château de Versailles sans faire la queue et sans avoir à acheter à l'avance des billets coupe-file. Mais, en même temps, je puis témoigner que, dès les années 1950 , les plages de la Côte d'azur étaient saturées dès $11 \mathrm{~h}$ du matin et que des embouteillages routiers qui n'avaient rien à envier aux bouchons actuels rythmaient la vie des autochtones comme des touristes. C'est qu'entre-temps, deux révolutions liées se sont produites : quand j'étais encore enfant, la Terre comptait 3 milliards d'habitants (en 1960) et le nombre des arrivées internationales de touristes était de l'ordre de 70 millions. Aujourd'hui, la population mondiale s'approche des 7,5 milliards d'habitants et le nombre des arrivées internationales a dépassé 1,2 milliard. II est évident que le nombre pèse de tout son poids sur les ressources de la Terre comme sur les lieux touristiques. Certains lieux gèrent cet afflux et en tirent des profits croissants (Benidorm, première station touristique espagnole, a ainsi dépassé le million de nuitées), d'autres le gèrent, en tirent des profits mais feignent d'en être victimes (Venise, avec ses 23 millions de touristes), tandis que d'autres encore en sont véritablement accablés (par exemple, les villages des Cinque Terre, en Ligurie, qui doivent faire face à un afflux qui désormais, en saison, paralyse la vie locale). L'explosion des fréquentations s'est accompagnée d'une profonde transformation des clientèles: les touristes japonais ont progressivement pris place dans le paysage des lieux touristiques au cours des dernières décennies du XX siècle; les touristes chinois sont désormais omniprésents partout dans le Monde: avec plus de 100 millions de touristes, il s'agit du premier marché émetteur. Et le mouvement se poursuit, à un rythme qui semble inexorable et qui est susceptible de saturer tous les lieux touristiques mondialisés de la planète: il suffit d'un reportage télévisé pour que des Chinois qui n'avaient jamais quitté leur pays auparavant aient par exemple l'idée d'aller voir les Cinque Terre $^{5}$... Bref, en très peu de temps, le paysage touristique de la planète a encore changé.

C'est dans ce contexte qu'il faut placer les objectifs comme les nécessités d'un tourisme dit "durable ", mais à la condition de ne pas se payer de mots et d'éviter de penser que la recherche de la « durabilité » n'engendrerait que des processus univoques et vertueux.

\section{LES DIFFICULTES DE MISE EN CEUVRE D'UN TOURISME DURABLE}

\subsection{Des discours aux actes}

Le " tourisme durable " est un concept apparu dans les discours, puis laborieusement mis en actes. L’idéologie a donc précédé les mises en œuvre, les- quelles peuvent se décomposer en bonnes intentions (chartes de bonne conduite, campagnes d'incitation à la limitation de la consommation d'eau, etc.), en classements de nature administrative (espaces protégés, de réserve, etc.) et en réalisations concrètes, à des échelles très diverses (des hôtels HQE aux « véloroutes " qui parcourent plusieurs régions). Tout cela compte, à l'échelle de chaque réalisation ou de chaque changement de pratique, les petits ruisseaux faisant les grandes rivières. Mais, une grande partie de ces mesures ne fait que limiter certains effets néfastes ou pervers dans des lieux déjà totalement transformés, sociologiquement et écologiquement, par l'intensité des aménagements et des fréquentations. Autrement dit, il s'agit alors de correctifs destinés à sauver ce qui pourrait l'être encore et/ou à permettre à ces lieux de continuer à fonctionner, voire d'accroître leur rendement. Par ailleurs, on sait bien que certaines mesures de classement (y compris, au plus haut niveau, au patrimoine de l'humanité par l'UNESCO) visent à la fois, dans une parfaite contradiction, à une meilleure protection et à une médiatisation plus importante dont on attend, avec un complet cynisme, une fréquentation encore renforcée.

\subsection{Des parades inégalement efficaces face aux problèmes à résoudre}

Face à un afflux touristique croissant, les lieux s'organisent, avec plus ou moins d'efficacité, afin de tenter de faire cohabiter les contenus contradictoires du développement durable. La plupart des lieux sont engagés dans la politique du toujours plus, qu'il s'agisse d'atteindre à des taux d'occupation plus élevés ou de renforcer encore les capacités d'accueil. Quelquesuns se trouvent dans des situations de quasi blocage, lorsque les flux en viennent à les saturer, au moins temporairement. Pour apprécier les politiques locales ou régionales de développement durable, il convient donc d'opérer une différenciation entre les types de lieux touristiques.

Très schématiquement, il est possible de distinguer entre quatre principales catégories :

La première rassemble les stations touristiques qui sont en train d'évoluer vers un modèle urbain puisqu'elles ont à gérer des flux de type urbain avec les inputs et outputs d'une ville.

Les conurbations touristiques constituent une deuxième catégorie, formée de petites, moyennes et grandes stations qui grandissent et tendent, sauf intervention forte et rarissime des Etats, à constituer des ensembles urbains plus complexes lorsqu'elles 
s'affranchissent de fonctionnements saisonniers et attirent d'autres populations et d'autres activités que touristiques.

A terme, la première et la deuxième catégorie tendent à se rapprocher des grandes villes et métropoles qui, presque toutes désormais, ont une fonction touristique importante (affaires et loisir). Elles ont à affronter et résoudre des problèmes de développement durable de type urbain, avec toutes les problématiques liées (qualité énergétique de l'habitat, traitement des eaux et déchets, place des écosystèmes naturels, etc.). Certains lieux de ces catégories se heurtent à des situations de saturation bien particulière : il s'agit des villages et petites villes touristifiés qui, à certains moments de l'année doivent gérer des afflux qui compromettent leur fonctionnement ordinaire (cf. l'exemple des Cinque Terre en Ligurie, déjà évoqué) ; pour rester en Italie, la côte Amalfitaine se heurte, par endroits et moments, aux mêmes saturations brutales, liées aux mêmes navires de croisière. Ces espaces de petite taille à fort pouvoir d'attraction sont amenés à mettre en place des politiques de quo- tas (c'est le cas des Cinque Terre en Italie ou du parc national d'Ordesa, dans les Pyrénées espagnoles), afin à la fois de tenter de protéger des éléments naturels menacés, la qualité de la visite touristique elle-même, ainsi que secondairement la qualité de la vie des autochtones ne vivant pas du tourisme.

Troisième catégorie, les espaces peu ou pas touristiques et non protégés constituent la cible des nouvelles stratégies liées à l'idéologie du développement durable, à la faveur de modes touristiques qui se veulent respectueuses des cultures autochtones (tourisme solidaire, tourisme responsable, tourisme alternatif, etc.) mais qui, de fait, sont les chevaux de Troie destinés à intégrer ces espaces naguère encore oubliés dans l'espace touristique mondialisé.

Enfin, une dernière catégorie rassemble les espaces naturels protégés où toute intervention touristique est en théorie prohibée. Dans la pratique, les fréquentations y sont en général autorisées - mais encadrées et parfois payantes - et leur pression sur l'environnement, même sans aménagement, n'y est

Figure 1. Débarquement des croisièristes du Seven Seas Explorer, à Amalfi (septembre 2016)

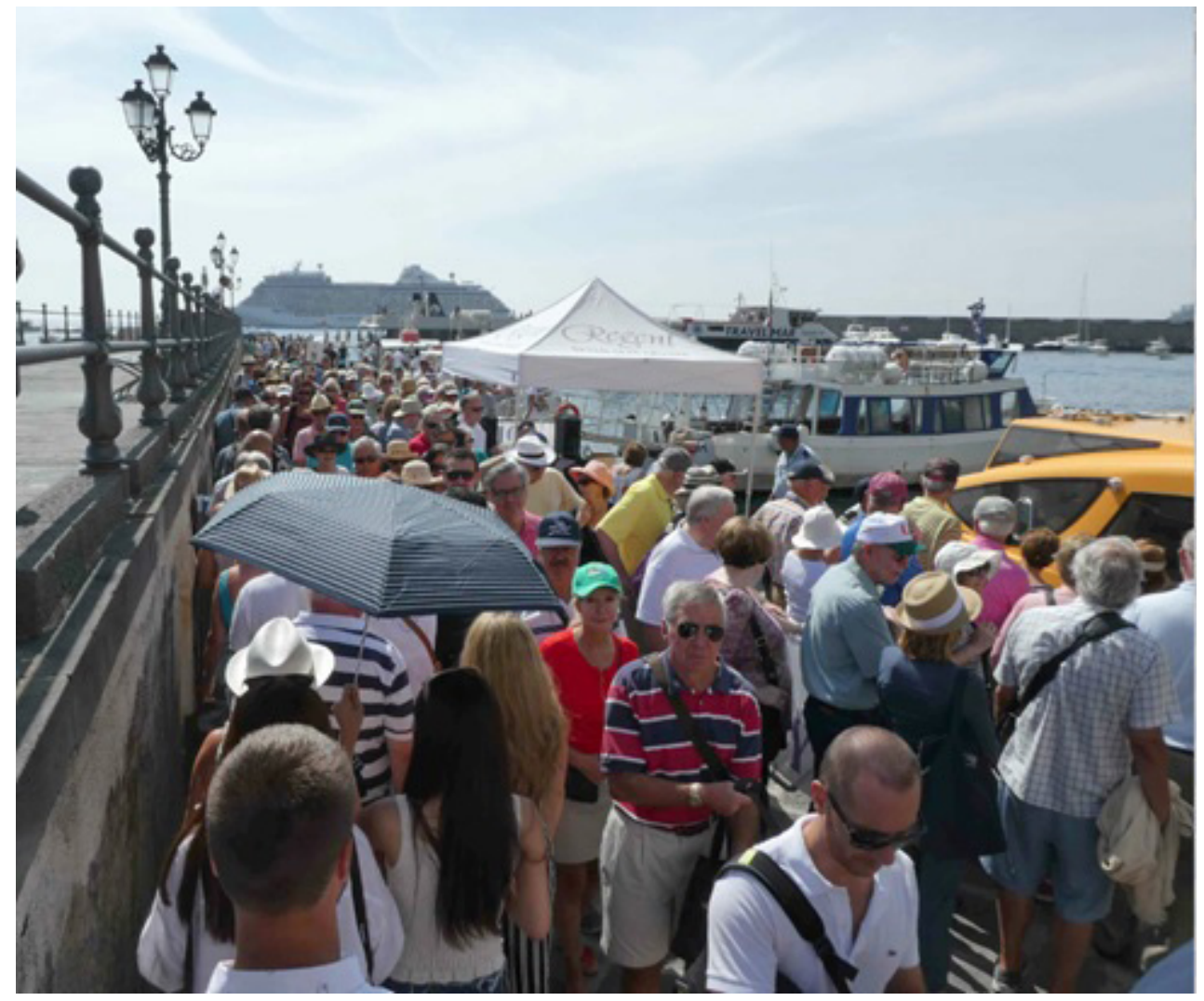

(c) R. Knafou. 
Figure 2. Trois navires de croisière (le géant Harmony of the Seas, au centre), à Naples (septembre 2016)

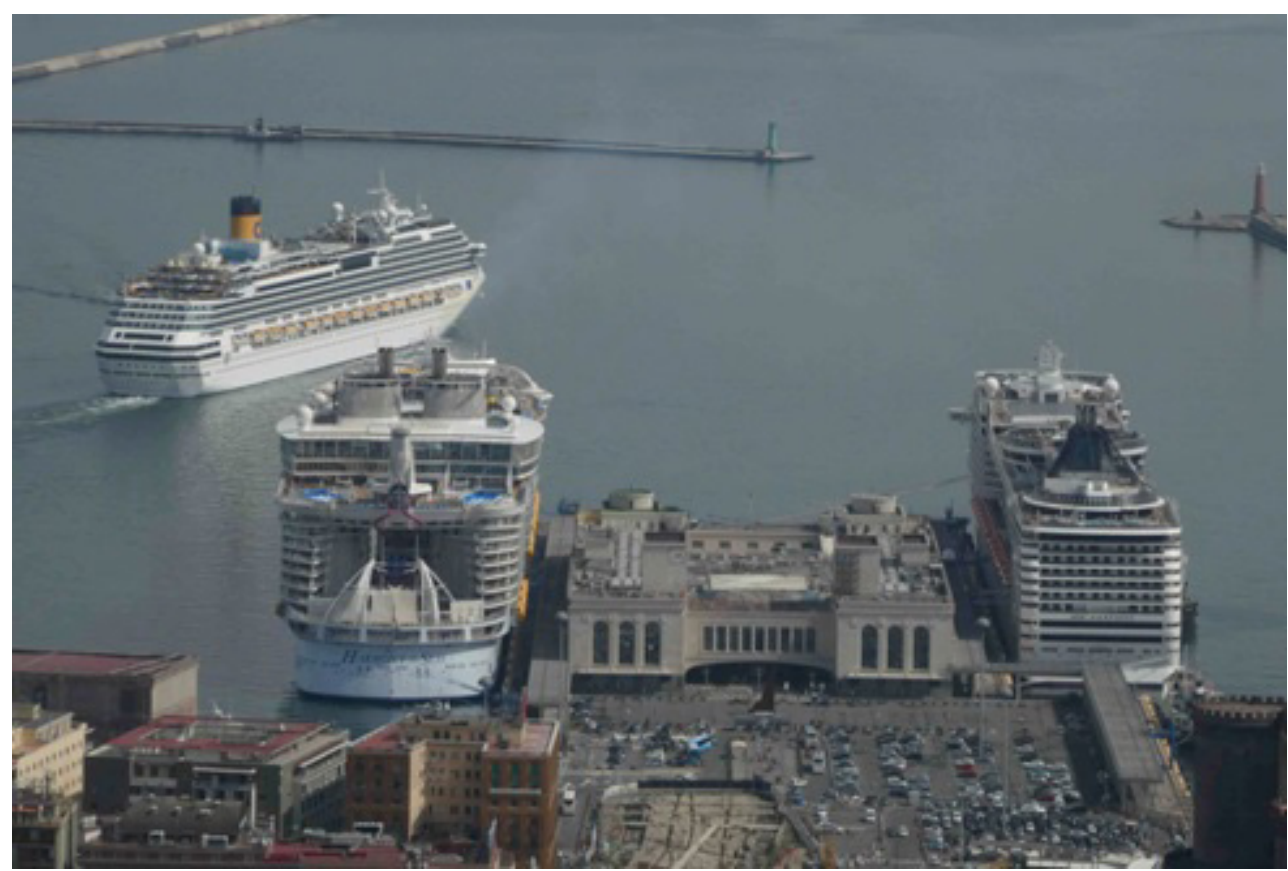

Il est évident que, dans les rues étroites d'Amalfi, le débarquement de 4000 croiséristes produit des effets infiniment plus perturbateurs que, le même jour, le mouillage de ces trois navires de croisère à Naples, avec un total d'environ 15000 passagers.

(C) R. Knafou.

pas négligeable. Seule une minorité d'espaces d'accès réellement interdits à toute fréquentation touristique constituent de véritables réserves d'espaces plus ou moins naturels ou renaturalisés.

Donc, quelles que soient les volontés politiques en faveur d'un " tourisme durable ", les dés sont jetés pour la plupart des lieux et espaces touristiques et les améliorations ne pourront se faire qu'à la marge, car les grandes concentrations touristiques continuent de bourgeonner, les touristes s'insinuent dans les espaces les plus reculés, avec la caution du « développement durable ${ }^{6}$ et une minorité d'espaces sont mis sous scellés (mais pour combien de temps ?).

Dans ces conditions, l'efficacité du tourisme durable est à relativiser fortement (même si l'analyse qui précède n'en minimise pas la nécessité). C'est dans ce contexte qu'il faut replacer le tourisme réflexif, puisque si agir sur les espaces est désormais à la fois coûteux et d'effet assez limité, il est possible d'agir sur les touristes et le système touristique dont ils constituent la raison d'être.

\section{LA VOIE D'UN « TOURISME REFLEXIF »}

Plutôt que de chercher à culpabiliser le touriste, en lui donnant mauvaise conscience, en l'infantilisant [exemples: " je ne prends pas de bains au Maroc ", "j'évite I' "American Breakfast » au Vietnam », « je fais attention à ne pas manger d'espèce en voie de disparition $\left.{ }^{7}\right]$, on peut préférer inviter le touriste et le système touristique à réfléchir sur ce qui se joue dans la pratique touristique comme dans la fréquentation de lieux touristiques, afin à la fois de les infléchir potentiellement et de faire de ses vacances un moment encore plus privilégié dans la construction de soi à travers la découverte de nouvelles altérités.

\subsection{Un concept né d'une convergence}

Le concept de " tourisme réflexif " est né de la convergence d'un parcours personnel, entre plusieurs décennies d'expérience professionnelle dans le champ de la recherche sur le tourisme comme de pratiques touristiques diverses et un engagement plus récent sur le terrain de la mémoire, dans le cadre du Mémorial du Camp des Milles, à Aix-en-Provence. Celui-ci, finalement inauguré en septembre 2012, n'est pas un mémorial comme les autres car, à côté des désormais classiques volets historique et mémoriel, il propose un troisième volet dit réflexif, intitulé «Comprendre pour demain ", qui constitue son aspect le plus novateur. L'objectif de la démarche est d'inciter le visiteur, après l'information acquise lors de la pre- 
mière partie de la visite et l'émotion suscitée par l'état de conservation du lieu d'internement ${ }^{8}$, à prendre conscience des mécanismes qui ont mené au crime de masse et, au-delà, à se mobiliser afin de tenter d'éviter que les engrenages identifiés ne conduisent à nouveau à des situations de crise et de catastrophe. L'originalité du projet est donc de ne pas se contenter d'évoquer classiquement et réthoriquement les "leçons de l'histoire ", mais de provoquer chez le visiteur une réflexion salutaire, porteuse d'une compréhension intime des situations et, partant, d'une prise de conscience afin d'être ou de demeurer un acteur agissant de son propre destin. Ma participation au travail sur ce "volet réflexif " du Camp des Milles a donc constitué un moment fondateur d'une réflexion sur les enjeux et les conditions d'une réflexivité appliquée à différentes pratiques sociales, parmi lesquelles le tourisme, mon champ de recherche conçu lui-même comme un enjeu de société d'importance moins anodine que celle dont beaucoup le créditent (Equipe MIT, 2005).

Cette convergence entre un champ thématique - en l'occurrence, d'abord la mémoire puis le tourisme - et une démarche réflexive s'inscrit dans un " tournant réflexif ", amorcé depuis plusieurs années et qui, au même moment, a suscité des applications dans d'autres lieux. En effet, l'esprit du temps, dans les lieux de mémoire, se contente de moins en moins de rappeler les faits fondateurs, mais prend appui sur ceux-ci pour aborder le contexte présent et même se projeter dans l'avenir. Ainsi, la démarche adoptée au Camp des Milles, en partie inspirée de celle mise en œuvres dans le Museum of Tolerance de Los Angeles, ouvert en 1993, est par exemple à mettre en parallèle avec l'initiative andalouse du Museo del Castillo de San Jorge, à Séville, où sur les ruines des bâtiments qui abritèrent pendant plusieurs siècles (entre 1481 et 1626 et entre 1639 et 1785) l'Inquisition espagnole, on a souhaité créer " un marco para la reflexión " (un cadre pour la réflexion). A partir du cas de I'Inquisition, il est donc proposé au visiteur de le dépasser et de réfléchir sur l'intolérance religieuse en général, les abus de pouvoir, mais aussi la pensée unique ou la déclaration universelle des droits de l'Homme. On notera que ce "centro temático de la tolerancia » (centre thématique sur la tolérance) a été pensé comme une " ressource touristique ", et inauguré par le Conseiller au tourisme de la Communauté autonome d'Andalousie, qui en a assuré $60 \%$ du financement. A notre connaissance, il n'y a pas eu de liens entre les deux initiatives, d'Aix-en-Provence et de Séville, le musée de San Jorge ayant été inauguré en décembre 2009, après huit ans de gestation ; seulement concomittance, témoignant que ces questions et questionnements étaient entrés dans la composition de l'air du temps, autrement dit que la société était en état de les recevoir, de les entendre, voire de les comprendre.

\subsection{La mémoire se prête particulièrement à la mise en œuvre d'un tourisme réflexif}

Il est évident que le terrain d'application premier et privilégié du tourisme réflexif est celui-là même qui a permis de le définir. Pour plusieurs raisons : d'abord, parce que la question de la mémoire, par définition, se prête, voire nécessite, une réflexivité car, sauf à se transformer en arme de propagande, elle nécessite à la fois de disposer d'une information sérieuse, de savoir faire le tri entre les connaissances et de chercher à se situer vis-à-vis des enjeux en cause. Ensuite, parce que nos sociétés se trouvent dans une situation apparemment paradoxale, car de plus en plus amnésiques ${ }^{9}$ et en même temps marquées par la multiplication des signes et lieux de mémoire. En fait, ce paradoxe n'est probablement qu'apparent : quand les repères fondamentaux s'effacent ou se diluent, on peut chercher à se raccrocher à quelques signes qui font sens ou, plus exactement, qui sont chargés de faire sens pour tout ou partie d'une population. Enfin, la multiplication des signes et lieux de mémoire engage le visiteur, le touriste, le citoyen, l'humaniste simplement curieux à ne pas seulement les recevoir ou les subir, à la différence de la visite d'un lieu de mémoire, laquelle nécessite une intention et une entrée dans un lieu fermé.

\subsection{Une première définition et enjeux}

Un tourisme réflexif est un tourisme qui s'interroge constamment sur lui-même, sa démarche, ses objectifs, ses finalités, ses moyens tout en se développant ; un tourisme qui considère le touriste en tant qu'être pensant, agissant ${ }^{10}$, actif, exigeant, conscient de ses choix et des conséquences qu'ils peuvent induire, concerné par la vie urbaine et la citoyenneté. Le tourisme réflexif vise à favoriser l'adoption par le touriste d'une posture réflexive et c'est l'adoption par le touriste d'une posture réflexive qui, par définition, permet de parler de tourisme réflexif, c'est-à-dire d'un système qui se tourne vers lui-même afin de mieux se connaître et mieux connaître sa capacité de réaction dans un contexte donné. Cette forme de tourisme est particulièrement adaptée à la ville, qui est un lieu de transformation permanente, de créations nouvelles ou renouvelées, qui à la fois suscite et permet des décryptages d'une réalité sociale complexe qui concerne, affecte le touriste, lequel est lui-même un 
urbain. Cette définition vaut pour les professionnels du tourisme comme pour les touristes eux-mêmes, ces deux catégories complémentaires ayant un intérêt commun à partager des mêmes valeurs.

Pour promouvoir un tel tourisme, trois types de raisons peuvent être mises en avant :

1. Fondamentalement, il s'agit de prendre en compte la maturité croissante du consommateur de tourisme, de plus en plus autonome, expérimenté et informé. A cet égard, la croissance exponentielle de l'information touristique désormais principalement alimentée par les touristes eux-mêmes via réseaux sociaux généralistes et communautés d'intérêts spécialisées (type Tripadvisor; notamment, Miguéns, Baggio et Costa, 2008; Vásquez, 2011) renouvelle profondément les conditions du développement du tourisme en général et favorise particulièrement l'émergence d'un tourisme réflexif, le touriste étant invité, en rendant compte publiquement de son expérience, à faire retour sur celle-ci.

2. Il s'agit d'un niveau supérieur d'une démarche-qualité, plus exigeante, démarche-qualité dans laquelle tous les lieux en Europe - dans un univers de plus en plus concurrentiel - sont désormais entrés. Mais cette démarche, pour ne pas être appliquée de manière mécanique, sans que les différents acteurs soient véritablement pénétrés de sa pertinence, nécessite la convocation de la réflexivité, à la fois source d'appropriation et d'approfondissement.

3. Stratégiquement, à une échelle nationale, il s'agit d'une étape de plus dans la quête de crédibilité du tourisme, encore trop souvent perçu par nos "élites" comme futile ou secondaire (probablement davantage en France qu'en Espagne).

Pour le touriste, il s'agit d'exploiter une potentialité qui existe chez chaque individu, mais selon des niveaux très différents selon l'âge, l'histoire personnelle, le type de formation, le niveau culturel, I'habitude ou non d'exercer ses capacités intellectuelles, son esprit critique, etc. Potentialité qui peut être plus facilement révélée/valorisée en situation de disponibilité physique et mentale créée par les vacances, le temps libre.

Ce souci de réflexivité va plus loin que le souci d'apprendre, de s'informer. L'information est généralement nécessaire pour alimenter la réflexion et celle-ci va à son tour réclamer davantage d'information : information et réflexion sont étroitement liées dès lors que le sujet porte sur une question qui intéresse la personne; mais selon des modalités qui gagneraient à être analysées de plus près dans le cas des apprentissages touristiques (en particulier, les conditions qui déclenchent l'intérêt, accrochent et motivent le visiteur). Quoiqu'il en soit, ce qui se joue avec la mise en avant d'une posture réflexive c'est sa capacité à faire évoluer sa pratique, par autocentrage plus que par simple réponse aux stimuli du marché ou aux bonnes paroles des discours institutionnels. Pour le comprendre, il est utile de faire un détour par quelques exemples de nouveaux objets urbains déclencheurs d'interrogations.

\subsection{Objets urbains furtifs et allusifs : exemples en Allemagne et en Espagne}

\subsubsection{Significations différentes des pavés de laiton dans l'espace public : les stolpersteine allemands et les pavés des juderías espagnoles}

Depuis un peu plus d'une vingtaine d'années, les pavés de laiton à visée mémorielle se sont plus ou moins subrepticement insérées dans les chaussées des villes européennes. Dès 1993, des "Stolpersteine » (littéralement, pierres d'achoppement, pierres sur lesquelles on peut trébucher), création de l'artiste berlinois Gunter Demnig, ont fait leur apparition dans des villes allemandes : ce sont des petits dés de béton ou de métal de $10 \mathrm{~cm}$ de côté enfoncés dans le sol, dont la face supérieure, affleurante, est recouverte d'une plaque en laiton qui indique sobrement le nom de la personne, sa date de naissance, la date de son arrestation et la date de son assassinat dans un camp d'extermination nazi. Plusieurs milliers de Stolpersteine ont ainsi été posées devant le dernier domicile des victimes du nazisme, principalement en Allemagne, mais aussi désormais dans d'autres pays européens. II s'agit donc d'objets destinés à entretenir la mémoire et sans vocation touristique. Mais, dès lors qu'ils font partie de la ville visitée, ils y participent malgré eux, d'autant plus que ces objets discrets, sur lesquels on peut marcher sans y prêter attention, sont intrigants pour qui les remarque. Le talent de leur concepteur a été de les rendre à la fois bien présents et peu visibles. Dans un monde où l'on met constamment les points sur les "i", G. Demnig a choisi de créer des objets d'une très grande sobriété, quasi furtifs dans l'espace dense et animé de la ville. Mais terriblement efficaces aussi dès lors qu'on les a repérés et qu'on en a compris la signification. Par leur insistante discrétion, 
ces stolpersteine ont une efficacité bien plus grande que les monuments aux morts ou les plaques commémoratives apposées sur les murs des immeubles. Ils confèrent à la ville un niveau particulier de lisibilité, notamment pour le visiteur étranger qui découvre à la fois un espace, ses vivants et ses morts.

Les pavés de laiton incrustés dans le sol de nombre de juderías d'Espagne ou du Portugal ont donc une toute autre signification que les Stolpersteine. On doit saluer la grande réussite graphique et esthétique de ces inscriptions qui réussissent à former avec les lettres hébraïques du mot " sefarad » (Espagne en hébreu) le contour schématique de la péninsule ibérique. Mais cette réussite dit bien aussi dans quel registre on se situe : celui de la communication touristique et peu celui de la mémoire, à proprement parler. En effet, ces pavés signalent au visiteur, au touriste qu'il se trouve dans une " judería " (" call », en Catalogne), c'est-à-dire un ancien quartier juif. Pendant plusieurs siècles, les juifs ont officiellement disparu de la péninsule ibérique, partis s'installer dans d'autres pays d'Europe, du bassin méditerranéen ou d'Amérique, ou convertis et enfouis dans des mémoires familiales souvent enterrées, mais parfois aussi entretenues sur la longue durée. Aujourd'hui, ces juderías à la signalétique flambant neuve témoignent du souci de certains Espagnols de se souvenir de leur passé juif, mais on pourrait discuter pour savoir s'il s'agit vraiment de " mémoire ", car celle-ci est portée par peu d'habitants et semble relever d'une stratégie essentielle- ment touristique ${ }^{11}$ visant surtout une clientèle ciblée, d'origine séfarade.

La limite du rapprochement entre les pavés de laiton allemands et espagnols tient aussi au très inégal éloignement temporel des faits qu'ils rappellent : sept décennies contre plus de cinq siècles. Toute la différence entre la mémoire et l'histoire, entre ce qui peut encore parler directement à une partie de la société et ce qui est à la fois très éloigné et peu approprié par la population d'accueil.

Car il faut rappeler que mémoire et tourisme ne font pas automatiquement bon ménage, à l'instar de cet exemple pris à Tolède, dans l'hypercentre touristique d'un lieu qui reçoit deux millions de visiteurs (touristes et excursionnistes, ces derniers venant de Madrid, distante de $70 \mathrm{~km}$ ) par an, avec une porte arabe précédée d'une statue de Cervantes, dont l'importance du lien avec la ville est sujette à discussion.

Les seules précisions qui sont données au visiteur sont une indication de nature architecturale (" porte arabe de la première enceinte militaire »), assortie d'un louable détail : "reconstruite" (elle a en effet été totalement détruite durant la guerre civile). Si on prend le soin d'apporter une précision sur l'authenticité de la porte, le nom de cette porte ("Arco de la sangre", l'arc du sang, appellation due à la confrérie de la Santa Sangre chargée d'assister les condamnée à mort lors de leur exécution), son nom est passé sous silence, de même que les massacres de juifs de

Figure 3. Stolpersteine, Fribourg-en-Brisgau (2012)

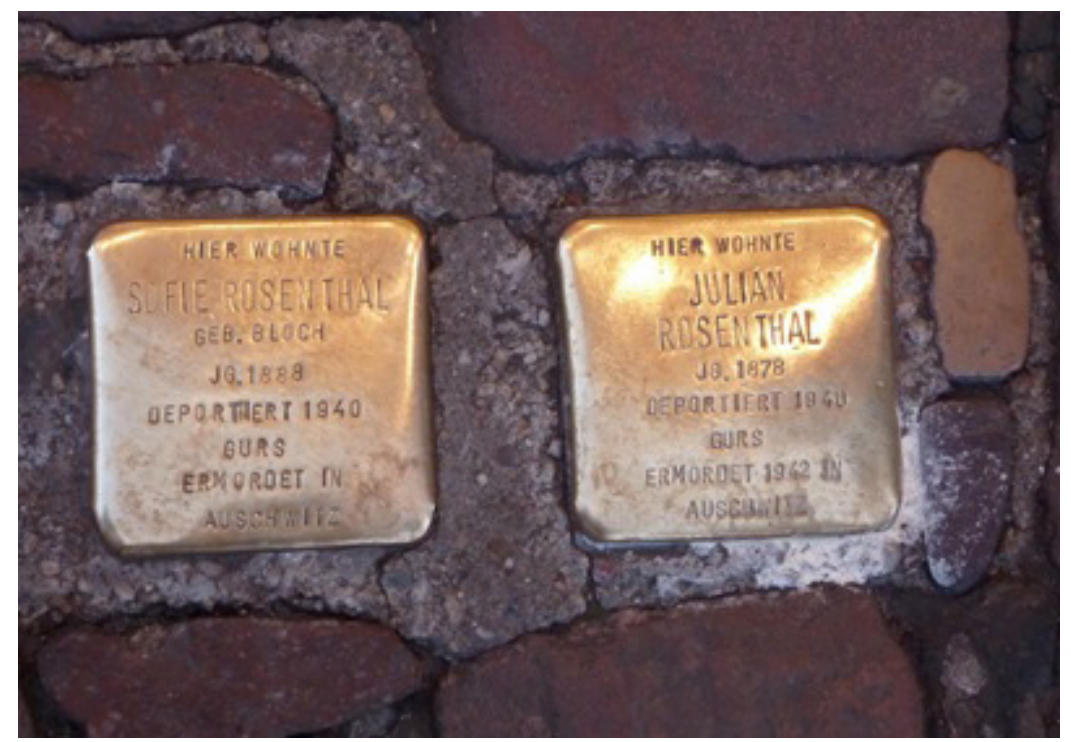

(C) R. Knafou. 
Figure 4. Dans les rues de la judería de Tarazona (Aragón, 2012)
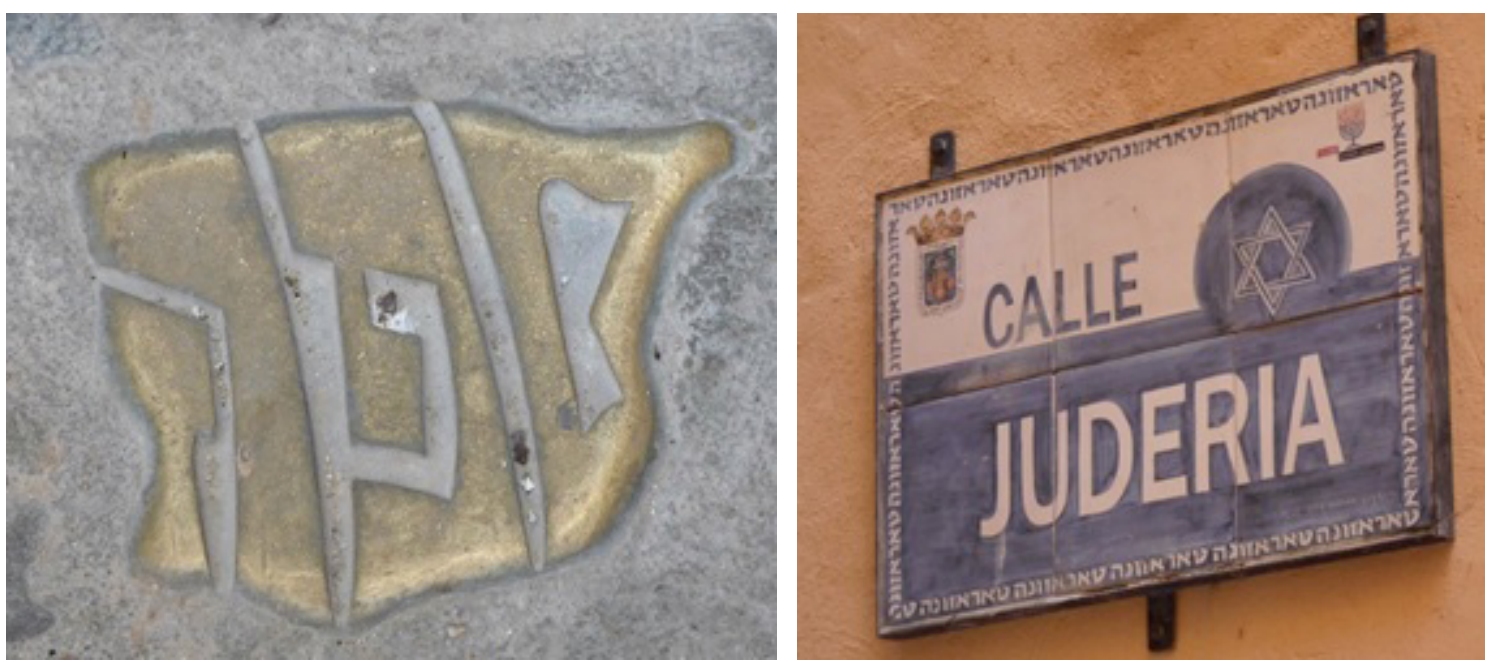

(c) R. Knafou.

Figure 5. El Arco de la sangre (Toledo, juin 2010)
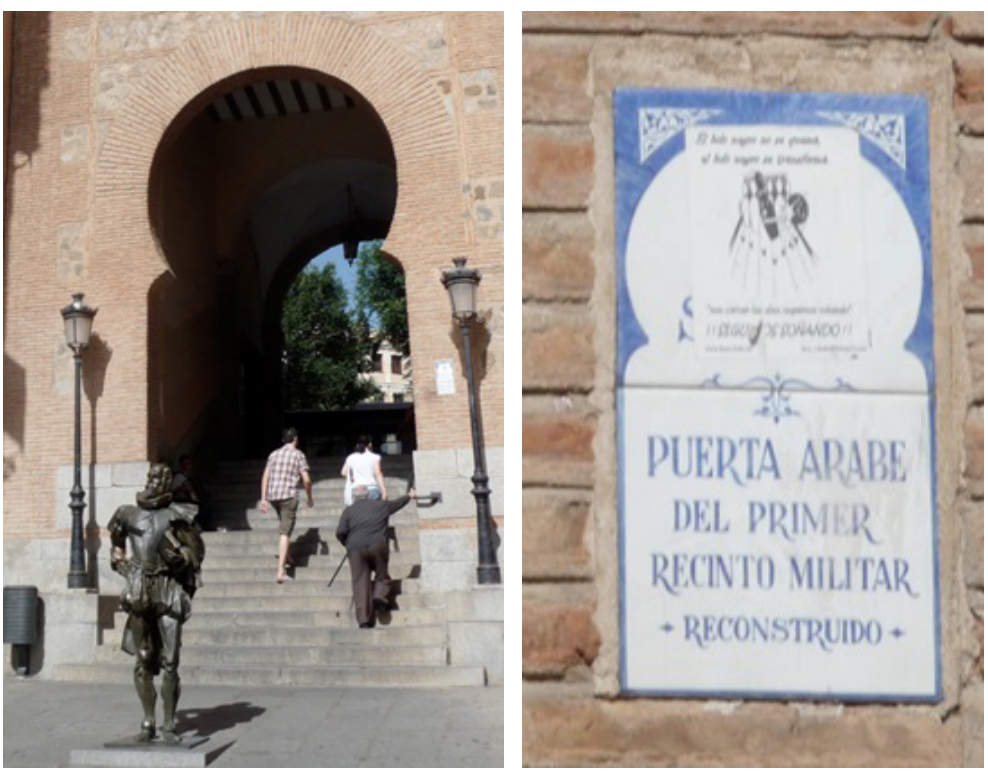

(C) R. Knafou.

1391 qui firent couler le sang dans ce même lieu (Cortanze, 2014). A Tolède, dans une ville ancienne où les traces du passé sont nombreuses, on souhaite éluder les épisodes difficiles, en préférant célébrer la diversité des trois cultures ("Le fait que chrétiens, Arabes et Juifs aient vécu ensemble pendant des siècles se reflète dans le patrimoine artistique et culturel de Tolède... ", http://www.spain.info/fr_FR/que-quieres/ ciudades-pueblos/otros-destinos/toledo.html), on trie et passe par pertes et profits la réalité d'une histoire terrible. L'histoire de la ville vue par les res- ponsables touristiques est une histoire frileuse et censurée. Sous couvert d'indications parcellaires relatives au passé de la ville et d'un désir d'une histoire consensuelle expurgée, nous sommes en présence de ce qui pourrait s'apparenter à un déni de mémoire ou à un désir de participation à l'écriture d'un nouveau roman national.

Quoi qu'il en soit, tous ces objets urbains partagent plusieurs points communs : ils se veulent des marqueurs de mémoire, ils sont furtifs, allusifs et, de ce fait, inégalement identifiés pour ce qu'ils sont, mais 
lorsqu'ils sont vus, car ils sont tout de même conçus pour être vus, ils sont destinés à susciter une démarche réflexive chez le passant, qu'il soit autochtone ou visiteur.

On en prendra un dernier exemple, pour illustrer le propos et mieux faire apparaître les enjeux : la Versunkene Bibliotek sur la Bebelplatz, à Berlin.

\subsubsection{La « Versunkene Bibliotek » à Berlin}

La Bebelplatz, à Berlin, face à l'université Humboldt, a été le 10 mai 1933 le lieu du premier autodafé nazi au cours duquel plus de 20.000 livres « d'esprit non allemand "(Heine, Mann, Marx, etc.) furent brûlés en présence de 70.000 personnes. Le souvenir de cet événement est discrètement rappelé par un mémorial connu sous le nom de "Versunkene Bibliotek » (la Bibliothèque vide) de l'artiste israélien Mischa Ullmann (1995) : une bibliothèque souterraine blanche aux rayonnages vides, visible à travers une plaque de verre encastrée au milieu des pavés de la place ; à distance, deux plaques de bronze évoquent l'événement, l'une d'elle portant un vers de Heinrich Heine : « Dort, wo man Bücher verbrennt, verbrennt man am Ende auch Menschen » ("Là où on brûle les livres, on finit par brûler les hommes ») [1823]. Sur cette vaste place nue, le visiteur peut sans peine passer à côté de ce marqueur de mémoire aussi discret qu'allusif : rien ne dépasse, il n'est pas visible de loin et seul le rassemblement fréquent de personnes regardant le sol peut inciter le curieux à s'approcher ; mais, de près, si le visiteur est saisi par l'étrangeté de la scène, rien n'indique sa signification, sauf s'il se déplace avec un guide touristique ou s'il connaît l'histoire du régime nazi. Cette plaque de verre livrant la vue de rayonnages blancs, nus et froids, remplit une de ses fonctions premières : car, en évoquant cet épisode glaçant de mai 1933 dont nous avons conservé films et photographies, elle intrigue le visiteur le plus étranger à l'histoire de l'Allemagne nazie. Et on sait que l'Homo sapiens se pose généralement des questions lorsqu'une situation l'intrigue, ce qui constitue le début d'un processus psychologique de portée difficile à prévoir.

\subsubsection{La "Säule der Toleranz" à Fribourg-en-Brisgau}

Fribourg-en-Brisgau est une ville prospère du riche Land de Bade-Wurtemberg, capitale de l'industrie solaire allemande, porte d'entrée touristique de la Forêt Noire (le cap du million de nuitées a été franchi dans la ville en 2007). La ville compte 230.000 habitants (630.000 dans l'aire urbaine). Surtout, Fribourg compte une université réputée, fondée en 1457, qui aujourd'hui est le premier employeur de la ville. Les bâtiments de l'Université se trouvent toujours pour l'essentiel dans la partie centrale de la ville ou à ses abords immédiats. Par conséquent, la population étudiante (plus de 33.000 personnes) fait partie du paysage urbain qu'elle anime, de jour comme de nuit. L'un de ses lieux favoris de rassemblement est l'Augustinerplatz, place piétonnière du centre, en légère déclivité, située à côté d'un biergarten. Les étudiants ont pris l'habitude de s'y retrouver en fin d'après-midi

Figure 6. Bebelplatz, devant l'université Humboldt, Berlin (décembre 2015)

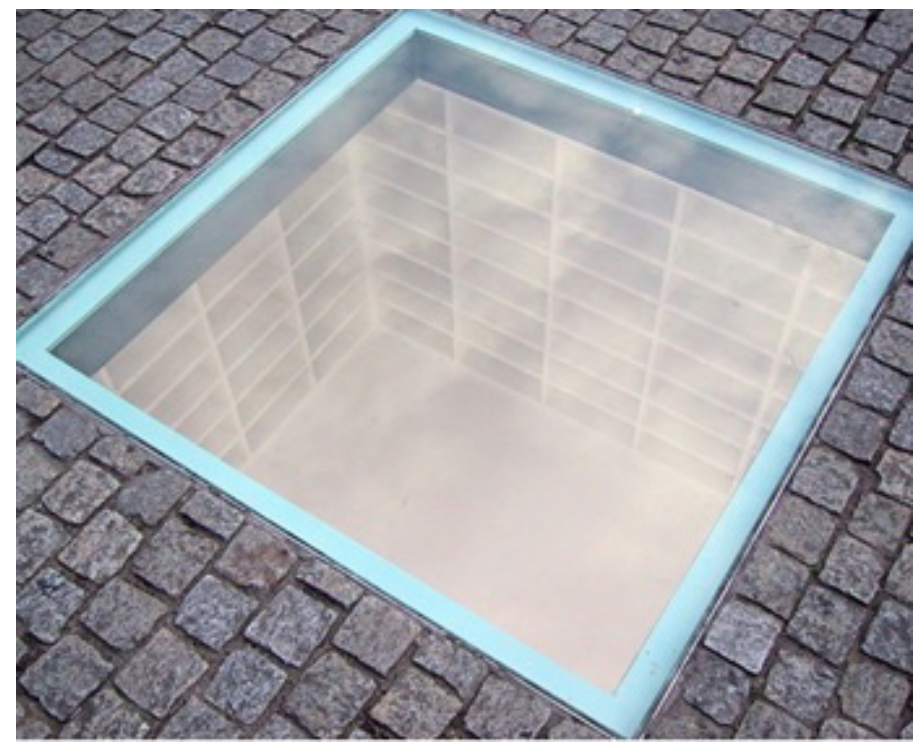

(C) R. Knafou. 
ainsi que les soirées de la belle saison; assis par terre, on y échange et on y boit. Comme les abords de cette place sont habités par des personnes qui n'apprécient pas nécessairement ces rassemblements estudiantins nocturnes et alcoolisés, les autorités municipales ont eu l'idée d'y installer en 2009 une "Säule der Toleranz" (colonne de la tolérance) qui présente la particularité d'enregistrer le niveau de nuisance sonore de l'animation suscitée par ces rassemblements et de l'indiquer par une couleur (en vert, le niveau est bas, en rouge, il est très élevé), avec l'effet escompté d'une prise de conscience et, en conséquence, d'une diminution du volume sonore. A une époque où, dans nos sociétés, on joue volontiers la carte de la prévention et du discours plutôt que de la répression, le dispositif est assurément intéressant. Et le touriste qui traverse cette place la nuit - le jour, le dispositif est éteint, et la colonne n'attire guère l'œil - en apprend plus qu'en un long discours sur la société allemande contemporaine et, en particulier, sur ses manières de gérer le vivre ensemble. Une telle initiative - quelle que soit son efficacité réelle, plus difficile à mesurer qu'un niveau sonore - ne peut donc qu'intéresser, voire interpeller le visiteur, lequel est généralement un urbain doté par définition d'une expérience des problèmes de voisinage et de nuisances sonores. Autrement dit, le touriste peut être autant intéressé par cet objet urbain que par les lieux du patrimoine qui l'entourent, tant il est vrai que les visites urbaines ne se limitent pas aux héritages du passé mais s'enrichissent et s'instruisent de toutes les nouveautés qui permettent la comparaison - et éventuellement, le transfert ou l'importation - avec la ville de résidence habituelle.

De ces quelques exemples récemment glanés en Europe on peut tirer quelques enseignements, à la fois sur les mutations des villes et sur l'évolution de leurs relations avec leurs visiteurs. La multiplication d'objets urbains de ce type constituent un champ nouveau d'analyses comme de mises en œuvre. On notera en particulier que ces OUFA (Objets urbains furtifs et allusifs) sont aux antipodes des centres d'interprétation qui les ont historiquement précédés. Rappelons qu'un centre d'interprétation est un lieu dont l'objectif est de valoriser un site par l'information et l'explication. On y trouve donc à la fois des informations sur le site visité ainsi que généralement la manière de le voir (dans les deux sens du terme). Le développement des centres d'interprétation est, non sans paradoxe, allé

Figure 7. La "Säule der Toleranz" à Fribourg-en-Brisgau

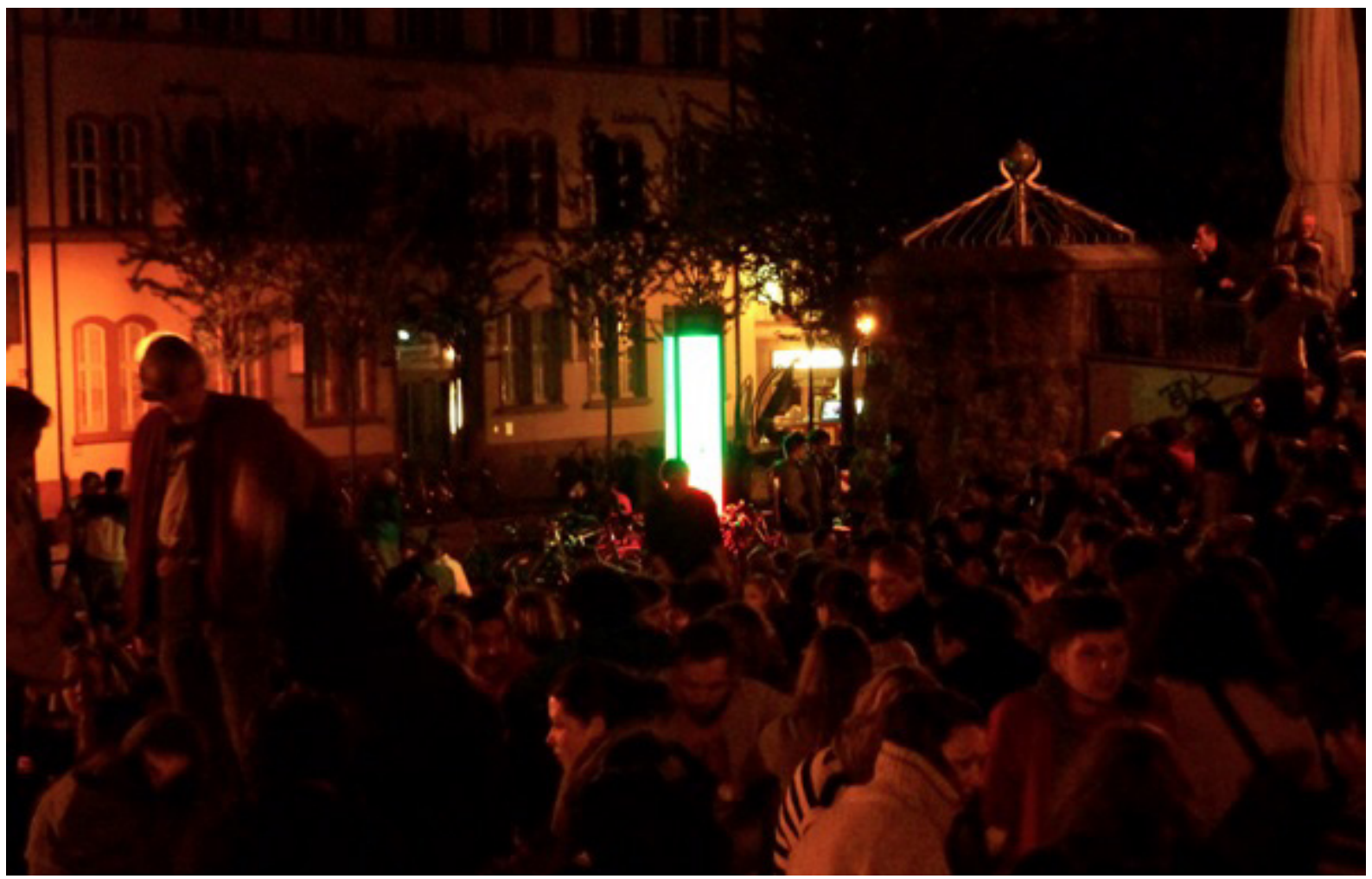

(c) R. Knafou, mai 2012. 
de pair avec le déclin de l’Ecole dans nos sociétés. Le problème que posent bien souvent les centres d'interprétation est qu'ils mêlent connaissances objectives et jugements de valeur, ce qui réduit la marge de manœuvre du visiteur. Les OUFA posent des questions, incitent à une recherche personnelle, tandis que les centres d'interprétation saturent l'intérêt.

\subsection{La ville touristique, réservoir à la fois de valida- tion de conformité et de sérendipité}

En tout état de cause, avec ces nouveaux objets urbains, il se joue un renversement des approches du tourisme. En effet, de plus en plus souvent, le touriste vient voir ce dont il a entendu parler et ce qu'il a déjà vu (au cinéma, à la télévision) et c'est du reste ce à quoi s'emploient les politiques de communication touristique, directe et indirecte. Il en résulte des flux massifs de touristes qui veulent vérifier par expérience directe, ce qu'ils ont déjà vu en images. Ce qui leur permet de réaliser des selfies de double validation conforme, pour ne pas dire conformiste: immortaliser un visage qu'ils connaissent intimement devant un paysage que tout le monde connaît ou reconnaît. En revanche, ces nouveaux objets urbains qui surprennent le visiteur rebattent les cartes en lui proposant des objets déconcertants, imprévus au point d'être parfois quasi invisibles mais qui, une fois approchés, libèrent leur potentiel de surprise et d'interpellation. Avec la diffusion de ces OUFA, la ville touristique renforce son potentiel de sérendipité (le fait de " trouver autre chose que ce que l'on cherchait ") et peut mettre en avant un tourisme s'appuyant sur une logique nouvelle permettant une lecture différente de la ville à visiter et à découvrir au sens plein du terme (combien de " découvertes " de lieux nouveaux constituent-elles réellement une découverte ?) Cela dit, cette phase n'aura, par définition, qu'un temps puisque le repérage de ces objets urbains, la diffusion de leur existence et de leur signification via les guides touristiques, les réseaux sociaux et les sites web collaboratifs aura pour conséquence d'atténuer ou de supprimer l'effet de surprise et de les reverser dans le champ des attractions recensées et évaluées. En attendant que surgissent d'autres objets nouveaux et déconcertants qu'appelle la fuite en avant de notre système touristique. Le surgissement, dans les lieux touristiques principalement urbains, d'objets inattendus conçus d'abord pour faire naître des questionnements, sources de culture et d'éventuelles remises en cause, entre dans la logique d'un tourisme réflexif, tout à la fois imaginatif, créatif et pas nécessairement très coûteux, dont les effets sur les personnes comme sur le système touristique méritent d'être étudiés, voire favorisés.

\section{CONCLUSION}

La gestion de la Terre en prenant en compte une dimension touristique qui s'affirme de plus en plus passe par quelques solutions classiques et moins classiques. L'interdiction totale de fréquenter des espaces naturels remarquables et analysés comme fragiles relève des solutions classiques, mais dont on ne peut faire l'économie, surtout dans la logique de transmettre à nos descendants des portions de surface terrestre intouchées. Par définition, ces espaces interdits ne peuvent être habités de manière permanente, afin de ne pas en faire des "zoos humains", de même qu'expulser des habitants pour mettre sous scellés une portion de territoire constituerait une solution inacceptable pour tout le monde. On a déjà évoqué la difficulté de gestion des espaces naturels à protéger mais peuplés et où les habitants voudront connaître une vie meilleure grâce à la venue de touristes : or, même si le nombre de touristes est limité et s'il s'agit de touristes bien intentionnés, solidaires, responsables, etc., on sait bien que lorsqu'on a mis le doigt dans l'engrenage il est généralement impossible d'en arrêter le processus. Nous sommes là en face de l'une des cibles stratégiques d'un tourisme durable aux effets potentiellement pervers. Dans les lieux du tourisme de masse, les dés sont jetés et les correctifs ne se feront qu'à la marge et progressivement, dans le cadre d'actions de moyen et long terme. C'est dans ce contexte qu'il faut placer le rôle d'un tourisme réflexif dont la contribution sera d'autant plus utile qu'il ne faut pas oublier que les clientèles touristiques sont volatiles et changent facilement de type de lieux selon les moments de l'année, les âges et les générations: ainsi, un même touriste peut fréquenter les plages de Benidorm en juillet et avoir une pratique éco-touristique dans des lieux peu accessibles à d'autres moments. C'est en pensant à cette diversité de pratiques d'un même touriste qu'il faut concevoir le recours à la réflexivité comme l'un des moyens de modification de ces mêmes pratiques sur le moyen et long terme. C'est ainsi que le tourisme réflexif pourra être l'un des fondements d'un tourisme durable désireux de dépasser les discours et de s'inscrire dans l'horizon d'un tourisme soucieux de mieux se comprendre et, ainsi, de mieux comprendre les autres. 


\section{NOTES}

1. Devenue ultérieurement «Mobilités, Itinéraires, Tourismes » mais, dès sa fondation, le tourisme était clairement son objet central de recherche, faisant de cette équipe la première en France ayant choisi de se consacrer à cette thématique.

2. Note à l'intention de lecteurs étrangers : cette expression française signifie réussir sans le savoir, sans dessein. Monsieur Jourdain est le principal personnage de la pièce de Molière, "Le bourgeois gentilhomme "; pour s'initier aux bonnes manières, il prend notamment les cours d'un maître de philosophie qui lui apprend ainsi qu'il pratique la prose sans le savoir : «Par ma foi, il y a plus de quarante ans que je dis de la prose sans que j'en susse rien, et je vous suis le plus obligé du monde de m'avoir appris cela. » (acte II, scène VI).

3. L'espagnol n'a pas commis cette erreur, en employant les mots "sostenible " ou « sustentable ».

4. La précision sémantique nécessaire dès lors que l'auteur est français - ayant été apportée en introduction, je précise que j'emploierai dans le reste de ce texte le mot "durable " dans son acception de "soutenable " (sostenible, en espagnol).

\section{BIBLIOGRAPHIE}

Cerda, Alexandre de la (2006). Couleurs de Biarritz. Pau: Éditions Sud Ouest.

Cortanze, G. de (2014). L'an prochain à Grenade. Paris: Albin Michel.

Equipe MIT (2005). Tourismes 1: lieux communs. Paris: Belin.

Equipe MIT (2011). Tourismes 3: la révolution durable. Paris: Belin.
5. Il s'agit de cinq villages de la côte ligure, classés au patrimoine mondial de I'UNESCO depuis 1997 et littéralement submergés par la fréquentation touristique depuis que les navires de croisière ont décidé d'y mouiller.

6. Et avec la complicité active des grandes collections de guides touristiques qui ont fait de la dénonciation du tourisme de masse leur fonds de commerce et multiplient les titres vers les destinations jusque-là les moins fréquentées, mais souvent les plus fragiles.

7. Il s'agit de préconisations trouvées sur le site du ministère français de l'Environnement : http://www.developpement-durable.gouv.fr/Comment-voyager-responsable.html

8. Entre septembre 1939 et décembre 1942, le Camp des Milles a été, sur le site d'une usine désaffectée pour cet usage, un camp d'internement et de déportation d'étrangers, d'antifaschistes, puis de plus de 2000 Juifs envoyés à Auschwitz.

9. Les historiens sont amenés à faire dans différents pays des constats similaires, lorsqu'ils constatent la double crise de l'enseignement de l'histoire à l'école, faite, d'une part, du recul des connais-

Miguéns J., Baggio R. et Costa C. (2008). Social media and Tourism Destinations: Tripadvisor Case Study. Proceedings of the IASK International Conference on 'Advances in Tourism Research'. Aveiro, Portugal, may 26-28, pp. 1-6. Disponible en: http://www.iby.it/turismo/papers/ baggio-aveiro2.pdf sances historiques en général et, d'autre part, de la perte de crédibilité, dans chaque pays, du " roman national » en raison du caractère de plus en plus composite de leur société.

10. En tant que chercheur, j'ai toujours travaillé sur la piste d'un touriste agissant et non " agi » comme une certaine littérature " scientifique " tendait à le montrer. J'en tire aujourd'hui de nouvelles conséquences, en vue de contribuer à améliorer la qualité de la visite touristique, la qualité de la relation entre le visiteur et le visité, entre le touriste et la société d'accueil, entre le touriste et les professionnels qui l'accueillent.

11. Cela dit, il convient de rappeler que le Congrès espagnol a récement (2015) adopté à l'unanimité une loi permettant aux descendants des juifs expulsés d'Espagne par les rois catholiques en 1492 d'obtenir facilement la nationalité, pour réparer " une erreur historique », cinq siècles plus tard.
Vásquez C. (2011). Complaints online: The case of TripAdvisor. Journal of Pragmatics, 43, 6, pp. 1707-1717. https://doi. org/10.1016/j.pragma.2010.11.007

Violier P. (2007). Tourisme et médias: regards d'un géographe. Le Temps des médias, 1, 8, pp. 159-170. https://doi. org/10.3917/tdm.008.0159 